\title{
The Pediatric Research Equity Act Moves Into Adolescence
}

\section{Citation}

Bourgeois, Florence T., and Thomas J. Hwang. 2017. “The Pediatric Research Equity Act Moves Into Adolescence.” JAMA 317 (3) (January 17): 259. doi:10.1001/jama.2016.18131.

\section{Published Version}

10.1001/jama.2016.18131

\section{Permanent link}

http://nrs.harvard.edu/urn-3:HUL.InstRepos:33749490

\section{Terms of Use}

This article was downloaded from Harvard University's DASH repository, and is made available under the terms and conditions applicable to Other Posted Material, as set forth at http:// nrs.harvard.edu/urn-3:HUL.InstRepos:dash.current.terms-of-use\#LAA

\section{Share Your Story}

The Harvard community has made this article openly available.

Please share how this access benefits you. Submit a story.

Accessibility 


\section{The Pediatric Research Equity Act Moves Into Adolescence}

Florence T. Bourgeois, MD, MPH

Computational Health Informatics Program, Boston Children's Hospital, Boston Massachusetts: and Department of Pediatrics, Harvard Medical School, Boston, Massachusetts.

Thomas J. Hwang, AB Computational Health Informatics Program, Boston Children's Hospital, Boston, Massachusetts; and Division of Pharmacoepidemiology and Pharmacoeconomics, Department of Medicine, Brigham and Women's Hospital, Boston, Massachusetts.

\section{Corresponding} Author: Florence T. Bourgeois, MD, MPH, Division of Emergency Medicine, Boston Children's Hospital, 300 Longwood Ave, Boston, MA 02115

(florence.bourgeois @childrens.harvard .edu).
Children continue to be underrepresented as participants in clinical trials, limiting the evidence available to guide treatment decisions. Among new interventional trials registered on ClinicalTrials.gov in 2015 , only $6 \%$ of 19239 trials focused on children from birth to 17 years of age, even though this age group comprises about a quarter of the US population. As a result, clinicians frequently use medications tested in adults for the treatment of children and adolescents. In one study, rates of off-label prescribing were estimated to involve $85 \%$ of 57000 hospitalized children nationally. ${ }^{1}$ Without adequate evidence to support these interventions, children may be exposed to serious unintended harms. Notable examples include the off-label use of verapamil to treat children with supraventricular tachycardia (associated with hypotension and death) and the antimicrobial chloramphenicol administered to infants (leading to fatal circulatory collapse).

To improve the clinical study of medications in children, Congress passed in 2002 the Best Pharmaceuticals for Children Act, which grants sponsors an additional 6 months of market exclusivity in return for voluntarily performing US Food and Drug Administration (FDA)requested studies in children. The Pediatric Research Equity Act (PREA), passed in 2003, is a complementary, mandatory program that authorizes theFDA to require the study of a new drug or biologic in pediatric populations (defined as < 17 years of age). Under PREA, sponsors must submit data that assess the safety and effectiveness of a product in children or that justify the extrapolation of adult data to relevant pediatric subpopulations for the indications under review in adults. The act's requirements apply to new drug applications and biologics license applications, as well as supplements to these, including new indications and formulations. Although these studies are ordinarily required before approval, sponsors can request that the FDA defer or waivetheir PREA requirements incertain cases (Box).

The Pediatric Research Equity Act has the potential to provide pediatric labeling data at the time of entry of a new product to the market, thereby helping to prevent nonevidence-based use of new therapeutics in children. However, several reports, including from the Institute of Medicine (now the National Academy of Medicine), ${ }^{2}$ have suggested that the goals of the program have been diluted by exemptions, frequent study delays, and inadequate results reporting. Since its last reauthorization in 2012 through the end of 2015, 53 of the 137 novel drugs approved by theFDA required pediatric studies under the act. ${ }^{3}$ Only 7 of these 53 products had completed pediatric studies at the time of approval and entered the market with pediatric-specific labeling information. ${ }^{3}$

Policy makers are currently negotiating the reauthorization of the Prescription Drug User Fee Act, due to expire in September 2017, which governs the FDA's drug ap- proval process as well as the PREA program. As the program marks its 13th anniversary and with as many as half of drug labels still lacking any pediatric information, the PREA program should be updated to better generate and communicate evidence used in clinical practice.

Promoting Research on Rare Diseases

Policy makers should assess whether the exemptions granted by the FDA under PREA are adequately serving the needs of patients and physicians. Overall, approximately half of all drug and biologic approvals are exempted from pediatric study requirements. ${ }^{4}$ The FDA may waive pediatric studies if, for example, they would be impossible or highly impractical, such as for diseases exclusively affecting adults. Another notable exemption is for orphan therapies, defined as drugs intended to treat diseases that affect 200000 or fewer people in the United States. However, orphan drugs accounted for nearly half (21 of 45) of all drugs approved in 2015 and 129 of 375 new drug approvals between 2003 and $2015 .{ }^{5}$ The majority of rare diseases have their onset in childhood, and more than $55 \%$ of orphan drug approvals involve conditions that affect both children and adults, with nearly $20 \%$ of orphan drug approvals involving conditions that are exclusively pediatric. Because orphan drugs are exempted from PREA requirements, few of these drugs are subsequently studied in all relevant age subgroups. For example, tadalafil was approved in 2009 as an orphan drug to treat pulmonary arterial hypertension in adults and was exempted from PREA requirements. Tadalafil is now used to treat pediatric patients with this disease but continues to lack FDA prescribing guidelines for children.

Expanding PREA to include orphan drugs, which would require a statutory amendment through legislation, could help increase the evidence base for pediatric populations with rare diseases and would harmonize the FDA's requirements with those of other regulators. In 2007, when the European Medicines Agency adopted its own version of PREA, orphan drugs were not exempted from pediatric study requirements.

Incentivizing Timely Completion of Pediatric Studies Sponsors may request deferrals of their PREA requirements. For example, between 2004 and 2007, deferrals were granted in $55 \%$ (338 of 605) of new and supplemental applications. ${ }^{4}$ Additionally, these studies can be deferred repeatedly. According to the FDA, as of December 2015,186 of 332 (56\%) deferred studies were granted further extensions. ${ }^{6}$ One example is methoxy polyethylene glycol-epoetin beta, an erythropoiesis-stimulating agent approved in $\mathbf{2 0 0 7}$ for the treatment of anemia associated with chronic renal failure in adults. The FDA required that the sponsor conduct a dose-finding study to 
Box. Overview of the Pediatric Research Equity Act

Scope

New drug applications, biologics licensing, and supplements to applications must contain data that

Enable assessment of the safety and effectiveness of the drug or biologic for the claimed indication in all relevant pediatric subpopulations; and

Support appropriate dosing and administration of the drug or biologic in each relevant pediatric subpopulation; or

Justify the extrapolation of pediatric effectiveness from studies involving adults, if the disease course or drug action is sufficiently similar between adults and pediatric patients.

Waivers

The act does not apply to products for an indication for which orphan designation has been granted. In addition, the FDA can waive requirements for some or all pediatric age groups if Necessary studies would be impossible or highly impracticable because, for example, the disease occurs primarily inadult populations;

Evidence strongly suggests the drug ineffective or unsafe;

The drug or biologic does not represent meaningful therapeutic benefit over existing therapies for pediatric patients and is not likely to be used in a substantial number of pediatric patients; or Reasonable attempts to produce a pediatric formulation have failed.

Deferrals

The FDA can grant deferral of submission of required pediatric data if The drug or biologic is ready for approval for use in adults before pediatric studies are complete;

There is evidence to support the delay of pediatric studies until additional safety or effectiveness data have been collected; or

There is another appropriate reason for deferral.

In addition, sponsors may request an extension of deferrals.

identify the optimum starting dose in pediatric patients. After an initial deferral, the study, due to be completed within 2 years, is still pending, and the deadline for the pediatric report has been extended to 2017-nearly 10 years after initial drug approval.

In 2012, Congress passed the fifth reauthorization of the Prescription Drug User Fee Act, which included several provisions aiming to increase the timely completion of pediatric studies. Specifically, sponsors must submit pediatric study plans with any deferral requests. These study plans, which detail the planned pediatric studies, are subject to review by an FDA pediatric committee. The FDA was also granted the authority to publicly post noncompliance letters if a sponsor fails to meet certain deadlines for the completion of pediatric studies. The influence of these changes on study completion rates should be closely monitored. Legislators could further strengthen the incentive for timely completion of pediatric studies by amending PREA to direct serially noncompliant sponsors to provide funds for a third party, such as the National Institutes of Health $(\mathrm{NIH})$, to complete the required study. The NIH is already authorized to conduct voluntary pediatric studies under the Best Pharmaceuticals for Children Act if the manufacturer declines to do so.

\section{Improving Transparency and Communication}

Once pediatric studies are completed, the results should be made available, ideally through timely publication in peer-reviewed literature, where they are most accessible. However, publication rates for pediatric trials are poor. One analysis of 33 drugs with safety concerns identified in FDA-requested pediatric studies found that only 16 (48\%) of these trials were reported in peer-reviewed publications, and many reports did not accurately reflect the findings presented in the FDA reviews. ${ }^{7}$ Consequently, these valuable pediatric data may fail to inform treatment decisions at the point of care and may not be reflected in guidelines, meta-analyses, or other clinical reviews. Policy makers should consider modifying the statute to define full compliance with PREA requirements as public dissemination of study results, ideally not only on ClinicalTrials.gov but also through a peer-reviewed publication.

In addition to trial results, making study plans publicly accessible could accelerate pediatric research and inform patients and clinicians about the translation of medical research. Before approval, sponsors must submit detailed pediatric study plans to the FDA that include clinically valuable information, such as justification for extrapolation of adult data for pediatric labeling, or supporting data for exemption or deferral requests (eg, evidence indicating that a drug would be ineffective or unsafe in certain pediatric age groups). Despite the public health interest in disclosure of such information, it is unclear if the FDA has the statutory authority to disclose these data. Because it is unlikely to be included in a standard trial protocol, this information may therefore never be publicly available, even if the protocol is eventually posted on ClinicalTrials. gov or as part of a peer-reviewed publication. PREA should be amended to authorize the FDA to provide more detailed information on these pediatric study plans.

From thalidomide to improperly formulated sulfanilamide, the history of drug regulation has been shaped immeasurably by the serious harms to children caused by inadequately tested therapies. The passage of PREA marked an important chapter in efforts to improve clinical study of novel therapeutics in this vulnerable population. Modernizing the PREA program would help ensure continued progress toward equity for children in drug development.

\section{ARTICLE INFORMATION}

Conflict of Interest Disclosures: Both authors have completed and submitted the ICMJE Form for Disclosure of Potential Conflicts of Interest. Mr Hwang reports prior employment by Blackstone and Bain Capital, which have invested in health care companies. No other disclosures were reported.

\section{REFERENCES}

1. Czaja AS, Reiter PD, Schultz ML, Valuck RJ. Patterns of off-label prescribing in the pediatric intensive care unit and prioritizing future research. J Pediatr Pharmacol Ther. 2015;20(3):186-196.
2. Institute of Medicine. Safe and Effective Medicines for Children. Washington, DC: National Academies Press; 2012

3. Drugs@FDA: FDA approved Drug Products. http://www.accessdata.fda.gov/scripts/cder/daf. Accessed November 1, 2016

4. Retrospective review of information submitted and actions taken in response to PREA 2003. http://www.fda.gov/downloads/Drugs

/DevelopmentApprovalProcess /DevelopmentResources/UCM197636.pdf. Accessed October 10, 2016.
5. Novel drugs summary: 2015. http://www.fda.gov /Drugs/DevelopmentApprovalProcess /DrugInnovation/ucm474696.htm. Accessed September 6, 2016.

6. Pediatric study deferrals and deferral extensions. http://www.fda.gov/downloads /ScienceResearch/SpecialTopics /PediatricTherapeuticsResearch/UCM210954.pdf. Accessed October 10, 2016.

7. Benjamin DK Jr, Smith PB, Sun MJ, et al. Safety and transparency of pediatric drug trials. Arch Pediatr Adolesc Med. 2009;163(12):1080-1086. 